\title{
Les impacts sociaux de la rénovation urbaine à Bruxelles : analyse des migrations intra-urbaines
}

The social impacts of urban renewal: an analysis of intra-urban migrations in Brussels

\section{Mathieu Van Criekingen}

\section{(2) OpenEdition}

\section{Journals}

Édition électronique

URL : http://journals.openedition.org/belgeo/16092

DOI : $10.4000 /$ belgeo.16092

ISSN : 2294-9135

Éditeur :

National Committee of Geography of Belgium, Société Royale Belge de Géographie

Édition imprimée

Date de publication : 30 décembre 2002

Pagination : 355-376

ISSN : 1377-2368

Référence électronique

Mathieu Van Criekingen, «Les impacts sociaux de la rénovation urbaine à Bruxelles : analyse des migrations intra-urbaines », Belgeo [En ligne], 4 | 2002, mis en ligne le 31 décembre 2002, consulté le 11 février 2021. URL : http://journals.openedition.org/belgeo/16092 ; DOI : https://doi.org/10.4000/ belgeo.16092

Ce document a été généré automatiquement le 11 février 2021.

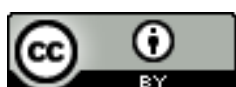

Belgeo est mis à disposition selon les termes de la licence Creative Commons Attribution 4.0 International. 


\title{
Les impacts sociaux de la rénovation urbaine à Bruxelles : analyse des migrations intra-urbaines
}

The social impacts of urban renewal: an analysis of intra-urban migrations in Brussels

\author{
Mathieu Van Criekingen
}

Je tiens à remercier Pierre Marissal pour son aide dans l'élaboration et la mise en œuvre de cette analyse, ainsi que Jean-Michel Decroly qui a encadré ma recherche.

1 Les politiques urbaines actuelles placent communément la problématique des quartiers urbains en difficultés dans la perspective du développement durable des villes (cf. le programme URBAN de l'U.E., p. ex.). Dans ce cadre, redévelopper l'attractivité résidentielle, commerciale ou économique de ces quartiers présenterait des avantages en termes de mixité sociale, de limitation de l'étalement urbain ou de réutilisation de bâtiments anciens délaissés (EGEC, 2000 p. ex.).

2 Cependant, les études consacrées aux dynamiques de rénovation de quartiers dégradés dans les villes occidentales ont souvent mis l'accent sur l'importance des impacts sociaux de ces dynamiques, particulièrement en termes de remplacement de populations. Classiquement, des groupes pourvus d'un important capital économique et/ou culturel réinvestissent des quartiers dégradés où habitent des populations pauvres et sans poids politique important; la revalorisation de ces quartiers y implique une élévation des coûts d'accès aux logements ou aux services de telle sorte que rénovation rime souvent avec éviction plus ou moins complète des populations initialement en place. L'abondante littérature (principalement anglo-saxonne) centrée sur la thématique de la gentrification a largement fait état de tels processus de remplacement de populations (LeGates \& Hartman, 1986; Smith \& Williams, 1986 ; Lyons, 1996 ; Smith, 1996 ; Atkinson, 2000 p. ex.).

Cette littérature laisse néanmoins très largement inexplorée la question de la destination des populations évincées. Seuls quelques rares travaux ont montré que les 
déménagements à courte distance paraissent prédominer. Cela a été interprété comme le reflet de l'ancrage des ménages urbains pauvres au sein de réseaux très locaux (réseaux de solidarités familiales ou "ethniques", réseaux d'échange ou d'entraide à l'échelle du quartier,...) (Lyons, 1996). A l'échelle de l'ensemble de la ville, ceci pousse à faire l'hypothèse que les processus de remplacement de populations dans les quartiers en voie de rénovation mènent à une surconcentration spatiale des populations socialement fragilisées : celles-ci sont contraintes de se relocaliser à proximité de leur ancien quartier, dans des portions du tissu urbain qui voient leur état de dégradation encore s'aggraver.

4 Les «bienfaits» et les "dommages» des dynamiques de rénovation urbaine ne paraissent donc pas équitablement répartis, ni socialement, ni spatialement. En contribuant à aggraver le fractionnement socio-spatial de l'espace urbain, et donc les tensions sociales dans la ville, ces dynamiques iraient même à l'encontre d'un modèle de développement urbain socialement durable. L'objet de cet article est d'étayer cette thèse sur base du cas bruxellois.

5 Au plan méthodologique, les différents aspects évoqués ci-dessus motivent à aborder la question des impacts sociaux des dynamiques de rénovation urbaine par le biais d'une analyse des migrations entre quartiers. Cette approche est très marginale dans la littérature, en partie sans doute à cause du peu de données migratoires intra-urbaines disponibles à une échelle fine. A Bruxelles, l'étude de De Lannoy \& De Corte (1994) fait exception. Menée à l'échelle des communes ${ }^{1}$, elle a mis en évidence un mouvement migratoire net de personnes de nationalité turque et (surtout) marocaine des communes du centre et de l'est de la Région de Bruxelles-Capitale vers les communes de l'ouest, Molenbeek en particulier, pour la période 1988-1992. Ce canevas appuie l'idée d'une surconcentration de populations socialement fragilisées dans la partie occidentale de la Région, plus populaire que les communes plus bourgeoises du quadrant sud-est de Bruxelles. De Lannoy \& De Corte (1994) ont suggéré que ce mouvement résultait de la croissance de la pression sur le marché locatif privé des communes centrales liée à la multiplication d'opérations de rénovation et au développement de la fonction tertiaire.

Cet article est basé sur un traitement original des données du dernier recensement publié (1er mars 1991) qui a permis de reconstituer les bilans migratoires de chaque secteur statistique bruxellois avec une commune de référence pour la période allant du 1er mars 1990 au 1er mars 1991. Malgré la petitesse de l'intervalle de temps imposé par la source de données, cette période d'un an est digne d'intérêt car elle se place pendant une vague de forte hausse des prix du logement à Bruxelles. Sur le marché locatif privé, segment dominant dans les quartiers centraux bruxellois (environ logements sur 3), l'écart entre le revenu des ménages et les coûts des logements s'est nettement creusé au début des années 1990 : les ménages bruxellois rangés dans le cinquième décile de revenu avaient accès à $55 \%$ du marché locatif privé en 1982 mais plus qu'à $42 \%$ en 1992. Cette contraction du marché locatif accessible à la moitié la moins nantie de la population bruxelloise s'est particulièrement fait sentir en 1990 et en 1991 (De Keersmaecker \& Carton, 1995). L'analyse de cette période de forte tension sur le marché du logement bruxellois est donc susceptible de faire ressortir des éléments structurels d'organisation des flux migratoires au sein de la ville.

7 La première partie de cet article est consacrée à détailler la méthode d'analyse. Ensuite, les résultats obtenus sont présentés. Ceux-ci permettent de démontrer, d'une part, 
l'existence de différents processus de remplacement de populations liés à différents types de rénovation urbaine ${ }^{2}$ (cas de Saint-Gilles et d'Etterbeek). D'autre part, ils appuient l'hypothèse d'une surconcentration de populations socialement fragilisées dans les portions déjà les plus dégradées et paupérisées de la ville suite au déploiement de dynamiques de rénovation dans les quartiers centraux (cas de Molenbeek et d'Anderlecht). Enfin, l'analyse du cas d'Ixelles permet d'éclairer la nature des itinéraires résidentiels qui alimentent certaines dynamiques de rénovation à Bruxelles.

\section{Méthode d'analyse : reconstitution des flux migratoires intra-urbains}

Mon objectif est de reconstituer les bilans migratoires de chaque secteur statistique de la Région de Bruxelles-Capitale avec une commune de référence donnée, à choisir parmi les 19 communes de la Région. Cela a pu être réalisé en trois étapes (figure 1).

Figure 1. Reconstitution des flux migratoires intra-urbains entre le $1^{\mathrm{er}}$ mars 1990 et le $1 \mathrm{er}$ mars 1991.

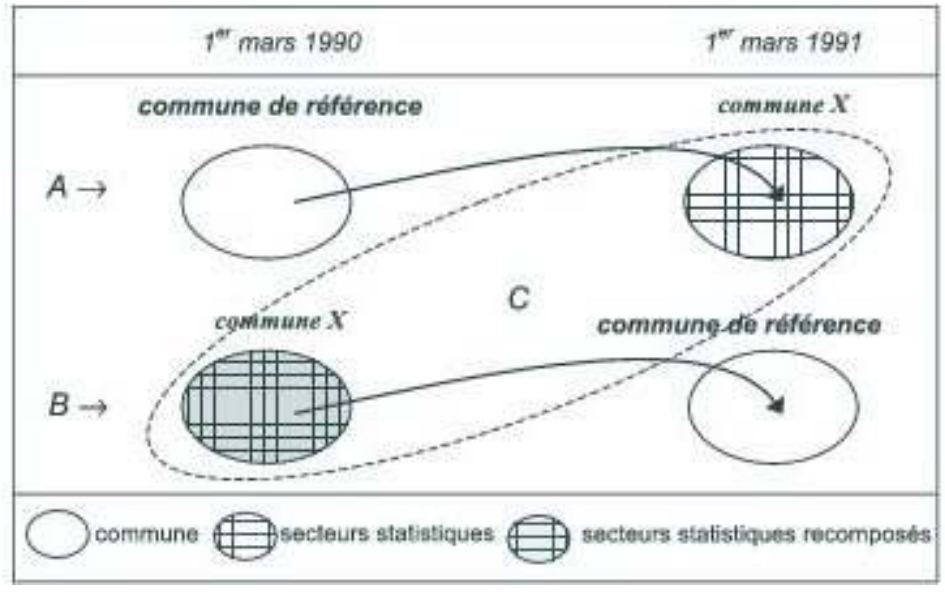

1. Le recensement de 1991 demandait de mentionner sa commune de résidence un an auparavant, soit le 1er mars 1990. Connaissant le lieu de résidence à la date du recensement, on peut calculer le flux de population ayant immigré vers chaque secteur statistique depuis une commune de référence donnée entre le 1er mars 1990 et le 1er mars 1991 (ligne A).

2. Dans le recensement, le flux de population ayant immigré vers chaque secteur statistique de la commune de référence n'est ventilé que par commune de provenance. Pour descendre à l'échelle des secteurs statistiques, il faut construire un modèle de répartition de ces immigrants dans les secteurs statistiques de départ (ligne B). Le modèle qui a été construit est basé sur des caractéristiques sociales spatialement discriminantes dans le contexte bruxellois : la nationalité pour les non Belges, le statut de chômeur (ou non) et le niveau de qualification ${ }^{3}$ pour les Belges. Concrètement, ceci signifie que l'ensemble des Anglais qui ont déménagé d'Uccle (commune $\mathrm{X}$ ) vers Saint-Gilles (commune de référence) entre le 1er mars 1990 et le 1er mars 1991 ont été répartis dans les secteurs statistiques d'Uccle proportionnellement au pourcentage d'Anglais dans chacun de ceux-ci. En ce qui concerne les enfants (1 à 18 ans) de nationalité belge, faute de pouvoir les distinguer par des caractéristiques socio-professionnelles ou de niveau de qualification, je leur ai affecté les caractéristiques de la personne de référence du ménage auquel ils appartiennent. Ceci est cohérent dans la mesure où les enfants migrent d'ordinaire avec leurs parents. 
3. En fin de compte, cette démarche permet de calculer le solde migratoire de chaque secteur statistique de la Région avec la commune de référence choisie (diagonale $\mathrm{C}$ ).

9 Cette démarche souffre de plusieurs limites qu'il importe d'avoir à l'esprit. D'une part, les données disponibles imposent le franchissement d'une limite communale pour qu'un déménagement soit pris en compte. Les flux migratoires intra-communaux sont par conséquent omis. D'autre part, une hypothèse de type gravitationnelle aurait pu être introduite dans le modèle de répartition des immigrants dans les secteurs statistiques de provenance de manière à rendre compte de la plus grande fréquence des migrations à courte distance (toutes choses étant égales par ailleurs). Ces deux points sont d'autant plus problématiques que la commune de référence est de grande taille (Bruxelles-Ville en particulier). Ils sont moins importants dans le cas des communes de petite taille (Saint-Gilles, Saint-Josse, Etterbeek,...). Enfin, cette démarche néglige également les mouvements multiples survenus entre les deux bornes temporelles fixées par le recensement ( $1^{\text {er }}$ mars 1990 et 1991). Tout porte cependant à croire que ces mouvements représentent très peu de choses.

10 Malgré l'intervalle de temps limité qu'impose la source de données mobilisée, les effectifs concernés sont importants: dans la Région de Bruxelles-Capitale, 114000 personnes de plus d'un an recensées au $1^{\text {er }}$ mars 1991 n'habitaient pas le même logement le 1er mars 1990 ( $12 \%$ de la population). Parmi eux, 74000 personnes résidaient dans une autre commune, dont 38600 dans une autre commune de la Région ${ }^{4}$. Mon analyse porte sur ces 38600 changements de commune enregistrés à Bruxelles entre le 1er mars 1990 et le 1er mars 1991. Ces flux ont été cartographiés par des triangles de surface proportionnelle au solde migratoire de chaque secteur statistique par rapport à la commune de référence choisie. Les triangles pointent vers le bas lorsque le mouvement net de population est dirigé vers la commune de référence (triangles gris), vers le haut lorsque le secteur statistique considéré reçoit plus de migrants provenant de la commune de référence qu'il n'y en envoie (triangles noirs). L'échelle des triangles est la même pour toutes les cartes.

11 Mon analyse est limitée au territoire de la Région de Bruxelles-Capitale. Il serait intéressant par ailleurs d'élargir ce cadre spatial pour inclure, par exemple, les communes du vieil axe industriel situées hors du territoire régional. On peut en effet supposer l'existence de migrations de populations évincées de quartiers centraux bruxellois vers de tels espaces dans la mesure où ceux-ci peuvent offrir certaines opportunités de logement à bon marché. De telles logiques sont parfois évoquées dans la presse à propos des conséquences de la «gentrification » du quartier des Marolles dans le Pentagone (Bokhorst, 2000).

\section{Rénovation urbaine et remplacement de populations à Saint-Gilles}

12 J'ai choisi Saint-Gilles comme première commune de référence pour le calcul des bilans migratoires (figure 2). Depuis le début des années 1980, de nettes dynamiques de rénovation résidentielle se sont déployées dans cette commune intercalée entre le quadrant aisé et le centre-ville. Activement soutenues par les pouvoirs publics locaux et régionaux (Decroly, Van Criekingen \& Crahay, 2001), elles ont principalement été nourries par l'installation de petits ménages de jeunes adultes, le plus souvent qualifiés, 
issus des classes moyennes et occupant une position transitoire dans le cycle de vie (entre décohabitation parentale et fixation familiale) comme sur le marché du travail (entre fin des études post-secondaires et stabilisation professionnelle). Même s'ils sont en moyenne plus aisés que la population en place, ces nouveaux habitants se distinguent encore davantage par leur capital culturel (Van Criekingen, 2001). Dans le même temps, le volume de population a baissé à Saint-Gilles tandis que le nombre de ménages augmentait. Cette diminution du nombre d'habitants pendant les années 1980 a été fortement tirée par la réduction des effectifs des nationalités représentatives des travailleurs immigrés à bas revenus, alors que le nombre d'étrangers augmentait dans toutes les autres communes bruxelloises (Marissal, 1994). Ces observations laissent à penser que les dynamiques de rénovation qui se sont déployées à Saint-Gilles pendant les années 1980 ont eu des impacts importants en termes d'éviction de populations socialement fragilisées.

Figure 2. Région de Bruxelles-Capitale: carte de repérage.

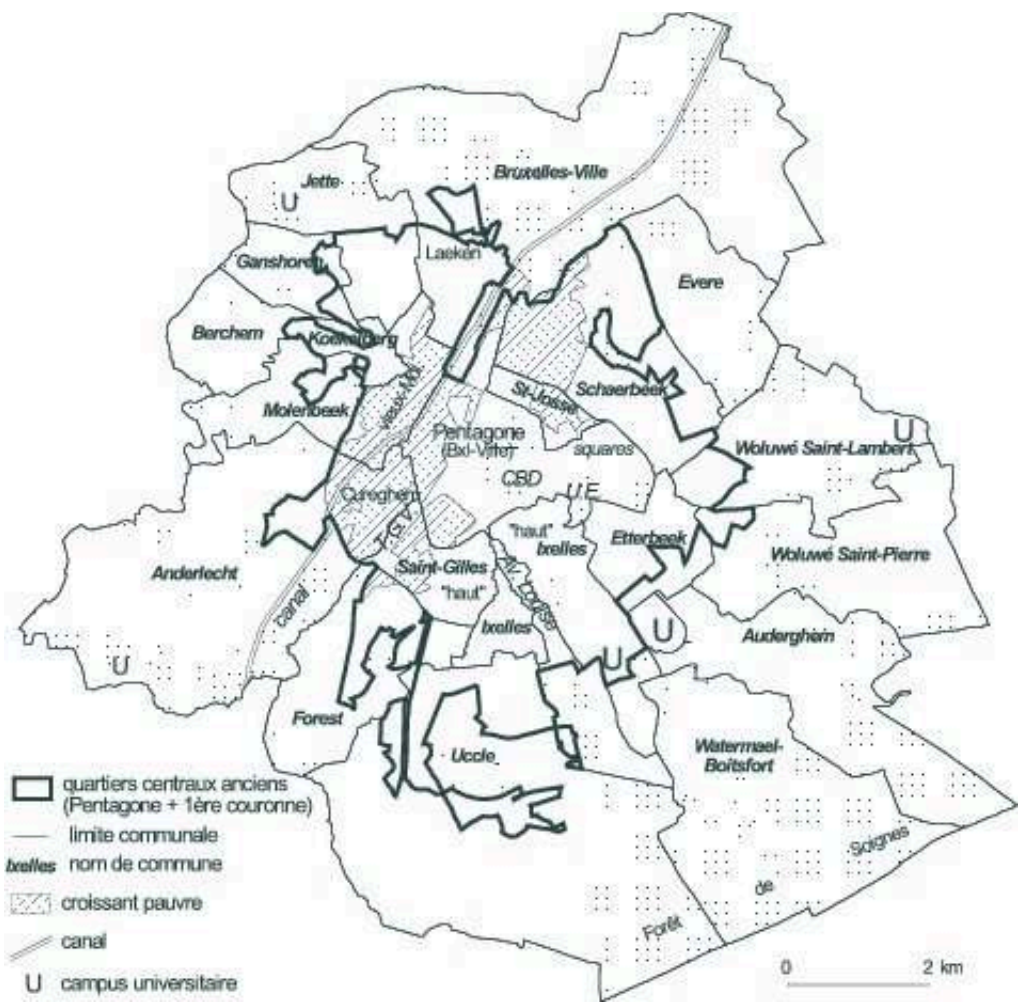

\section{Saint-Gilles : flux migratoires entre 1990 et 1991}

Entre 1990 et 1991, Saint-Gilles présente un solde migratoire très légèrement négatif avec l'ensemble des 18 autres communes bruxelloises (-171 habitants). Sous ce chiffre se cachent des mouvements de populations spatialement très contrastés (figure 3 ). Les flux entrants à Saint-Gilles proviennent du quadrant sud-est, d'Ixelles et d'Uccle en particulier, tandis que les émigrations depuis Saint-Gilles sont préférentiellement dirigées vers le centre de la ville (sud et ouest du Pentagone) et la partie occidentale de la Région (Cureghem et le long de l'axe du métro à Anderlecht, Molenbeek, Ganshoren, l'ouest de Schaerbeek, le bas de Forest et la partie occidentale d'Uccle). A première vue, ce schéma confirme l'hypothèse d'un remplacement de populations à Saint-Gilles : des 
groupes refoulés vers des espaces pauvres de la ville laisseraient la place à de nouveaux habitants issus de quartiers plus aisés.

Figure 3. Saint-Gilles : bilans migratoires 1990-1991 et 1980-1981.

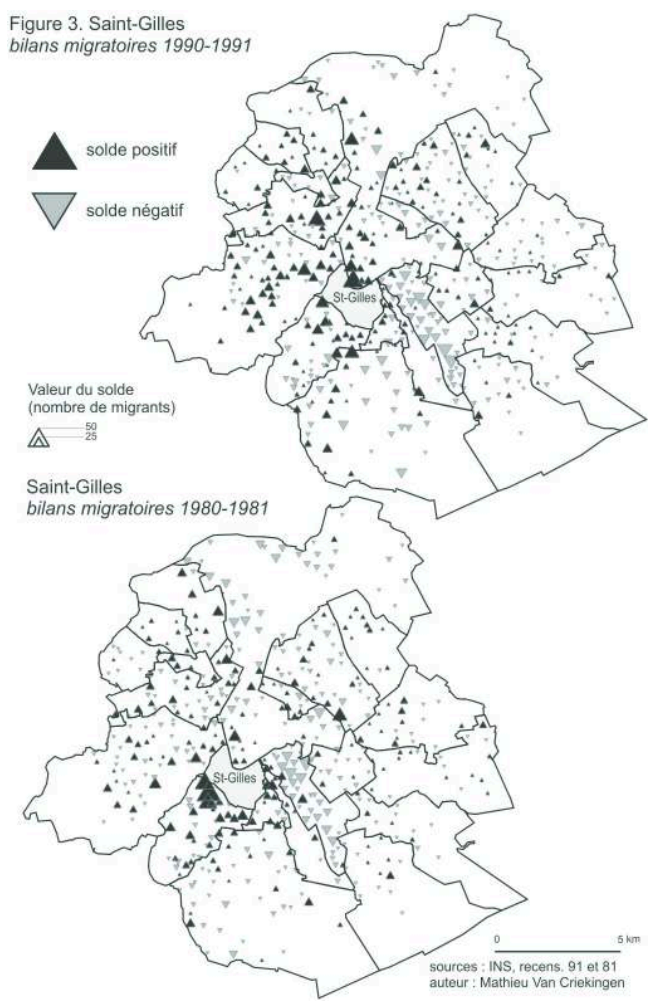

La confrontation de cette carte à celle portant sur la période $1^{\mathrm{er}}$ mars $1980-1^{\mathrm{er}}$ mars 1981, soit avant le plein développement des dynamiques de rénovation à Saint-Gilles, conforte cette idée (figure 3$)^{5}$. En effet, cette dernière présente un canevas spatial sensiblement différent. D'une part, les entrées à Saint-Gilles en provenance d'Ixelles étaient plus limitées à la partie orientale de cette commune (le haut de Saint-Gilles) et les échanges migratoires avec Uccle étaient nettement plus équilibrés. D'autre part, les émigrations de Saint-Gilles apparaissaient beaucoup plus limitées à une logique de desserrement à proximité, vers le bas de Forest particulièrement. Ce trait apparaît encore dix ans plus tard mais ne domine plus. Le canevas spatial observé entre 1990 et 1991 a donc été mis en place pendant les années 1980, alors que Saint-Gilles, sa partie haute en particulier, connaissait de fortes dynamiques de rénovation.

L'analyse des caractéristiques démographiques et socio-économiques des migrants de ou vers Saint-Gilles entre 1990 et 1991 permet de préciser le diagnostic.

\section{Saint-Gilles : contrastes démographiques}

Les structures d'âges des immigrants et des émigrants de Saint-Gilles présentent des profils très similaires (figure 4). Sans surprise, les classes d'âges les plus mobiles (0-4 ans et 20-29 ans) ressortent, tant parmi les Belges que parmi les étrangers ${ }^{6}$. Ceci traduit la coexistence de multiples logiques migratoires au sein de la ville, parmi lesquelles les flux croisés de populations occupent une place importante. Ces flux n'ont pas de signification particulière (déménagements entre quartiers semblables) et concernent 
logiquement les catégories d'âges les plus mobiles. Pour tester l'hypothèse d'un renouvellement social à Saint-Gilles, il est indispensable de percer l'écran de ces flux croisés de populations. Dans cette optique, j'ai d'abord isolé l'ensemble des émigrants de Saint-Gilles qui ont élu domicile, le $1^{\text {er }}$ mars 1991, dans un secteur statistique récepteur, c'est-à-dire pourvu d'un solde migratoire positif par rapport Saint-Gilles. Parmi ceux-ci, je n'ai pas repris les secteurs pour lesquels le bilan migratoire était quasiment nul, de manière à éliminer les cas de flux quasiment équilibrés ${ }^{7}$. De cette manière, 894 émigrants de Saint-Gilles sont repris, soit $36 \%$ de l'ensemble des émigrants de cette commune.

Figure 4. Structure d'âges des migrants par rapport à Saint-Gilles.

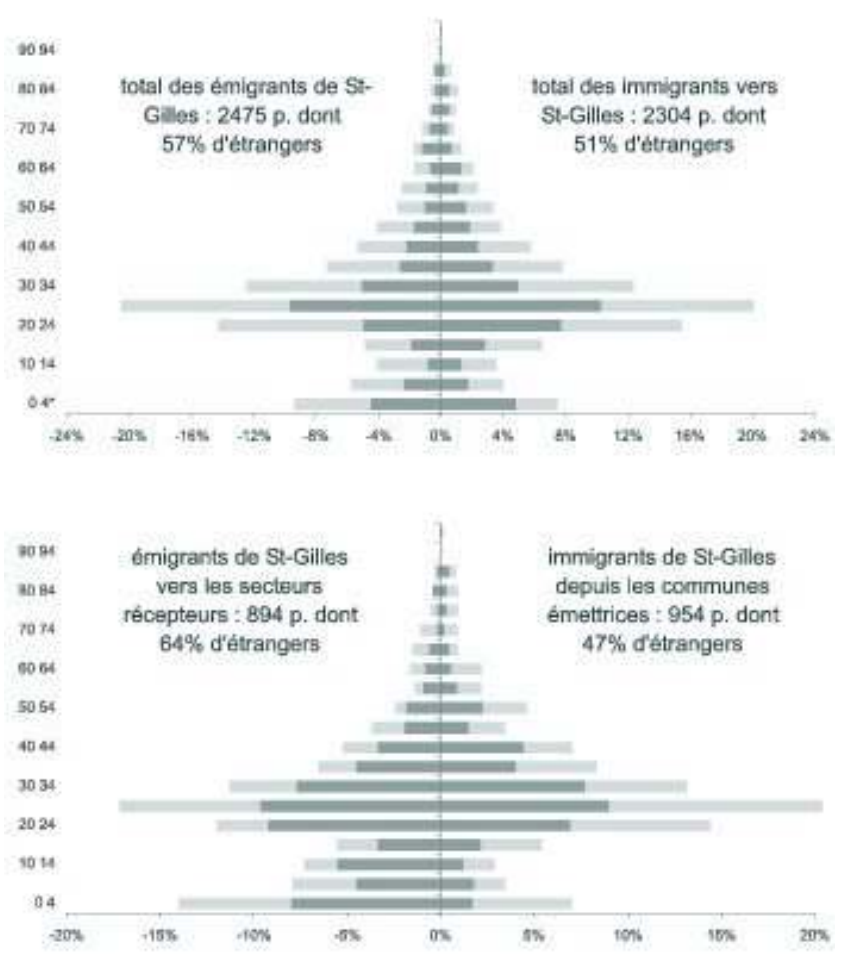

Les Belges à la naissance sont en clair, les étrangers, y compris les naturalisés, sont en foncé. Note: pour des raisons de traitement informatique, les pourcentages de la partie droite ont dû être notés en négatif; en réalité, il s'agit bien sûr de valeurs positives.

La structure par âge de cette sous-population (figure 4) présente un profil sensiblement différent de celui de l'ensemble des émigrants de Saint-Gilles: les enfants sont beaucoup plus représentés et les étrangers dominent largement. Il s'en dégage le portrait d'une émigration de grands ménages familiaux avec enfants d'origine immigrée. Ces ménages sont particulièrement vulnérables face aux dynamiques de rénovation des quartiers centraux. La combinaison de leur grande taille et de leurs faibles ressources financières les confronte au déficit de logements spacieux à bas prix sur le marché privé tandis que l'offre de logements sociaux (de grande taille ou non) ce type est largement déficitaire. Il y faut ajouter le poids des discriminations auxquelles doivent faire face ces ménages sans poids politique ${ }^{8}$ (Noël, 1998 ; Delbart, 1999).

Pour les immigrants arrivés à Saint-Gilles entre 1990 et 1991, les données sur lesquelles repose mon analyse imposent de travailler à l'échelle communale. J'ai donc dessiné la 
pyramide de l'ensemble des immigrants qui proviennent des communes qui ont un solde négatif avec Saint-Gilles (solde $\leq 5$ ). Il s'agit de Watermael-Boitsfort (39 émigrants vers Saint-Gilles, solde : -6), de Woluwé-Saint-Lambert $(43,-10)$, de Saint-Josse $(59,-14)$, d'Uccle $(265,-8)$ et, surtout, d'Ixelles $(448,-180)$, soit un total de 854 émigrants vers Saint-Gilles ( $37 \%$ du total), dont plus de la moitié provient d'Ixelles (figure 4). Ici, les Belges dominent les étrangers et la structure par âge cumule sur-spécificité des jeunes adultes et très faible représentation des enfants. Cette structure renvoie à un profil de jeunes ménages de petite taille (couples ou isolés, sans enfants), " post-étudiants » en provenance d'Ixelles surtout.

\section{Saint-Gilles : contrastes socio-économiques}

Ces différents groupes de migrants se distinguent nettement au plan socio-économique (tableau 1). D'une part, les émigrants de Saint-Gilles vers les secteurs récepteurs se caractérisent par des proportions élevées d'actifs peu qualifiés ou occupés à des professions manuelles (ouvriers, artisans,...) ou dans les services banals (horeca, services aux personnes,...). Ces catégories sont beaucoup moins représentées parmi les immigrants en provenance des communes émettrices. Ce sont cette fois les diplômés universitaires et les professions scientifiques ou libérales qui dominent. D'autre part, les nationalités représentatives des travailleurs immigrés à bas revenus (exYougoslaves, Turcs, Congolais, Algériens, Marocains et Tunisiens) sont près de six fois plus représentées parmi les émigrants de Saint-Gilles vers les secteurs récepteurs que les nationalités représentatives d'actifs internationaux de haut niveau (Ouesteuropéens, Etats-uniens et Japonais). Par contre, ces deux groupes de nationalités sont presque identiquement représentés parmi les immigrants vers Saint-Gilles en provenance des communes émettrices. 
Tableau 1. Caractéristiques socio-économiques des migrants de ou vers Saint-Gilles entre 1990 et 1991.

\begin{tabular}{|c|c|c|c|c|}
\hline & \multicolumn{2}{|c|}{$\begin{array}{l}\text { ÉMIGRANTS DEPUIS } \\
\text { SAINT-GILLS }\end{array}$} & \multirow{2}{*}{$\begin{array}{l}\text { IIMMIGRANTS VERS } \\
\text { SAINT-GILLS } \\
\text { total }\end{array}$} & \multirow[b]{2}{*}{$\begin{array}{l}\text { depuis les } \\
\text { communuse } \\
\text { au bilan } 2-5\end{array}$} \\
\hline & totol & $\begin{array}{l}\text { vers les } \\
\text { sect. stat. } \\
\text { au bilan } \geq 5\end{array}$ & & \\
\hline \multicolumn{5}{|c|}{ plus haut diplôme obtenu (\% dans I a population active) } \\
\hline universitare & $12 \%$ & $7 \%$ & $16 \%$ & $22 \%$ \\
\hline enseignement supérieur & $15 \%$ & $15 \%$ & $13 \%$ & $14 \%$ \\
\hline secondaire superieur & $19 \%$ & $15 \%$ & $18 \%$ & $19 \%$ \\
\hline secondaire inférieur & $17 \%$ & $19 \%$ & $17 \%$ & $16 \%$ \\
\hline primaire & $8 \%$ & $11 \%$ & $10 \%$ & $9 \%$ \\
\hline sans diplôme e tinconnu & $28 \%$ & $32 \%$ & $26 \%$ & $20 \%$ \\
\hline \multicolumn{5}{|c|}{ type de profession (\% dans la population active occupeé) } \\
\hline $\begin{array}{l}\text { professions scientifiques, } \\
\text { libérales et assimilés }\end{array}$ & $18 \%$ & $18 \%$ & $19 \%$ & $22 \%$ \\
\hline $\begin{array}{l}\text { chefs d'entreprise et } \\
\text { cadres supérieurs }\end{array}$ & $9 \%$ & $5 \%$ & $8 \%$ & $9 \%$ \\
\hline employés de bureau & $15 \%$ & $11 \%$ & $17 \%$ & $17 \%$ \\
\hline commerçants & $11 \%$ & $10 \%$ & $10 \%$ & $13 \%$ \\
\hline $\begin{array}{l}\text { ouviers, artisans, techniciens } \\
\text { et professions des transports }\end{array}$ & $27 \%$ & $32 \%$ & $27 \%$ & $19 \%$ \\
\hline travailleurs des services & $13 \%$ & $17 \%$ & $13 \%$ & $14 \%$ \\
\hline inconnus et autres & $7 \%$ & $8 \%$ & $5 \%$ & $5 \%$ \\
\hline \multicolumn{5}{|l|}{ nationolite à la naissance } \\
\hline \multicolumn{5}{|l|}{ ex-Yougoslave, Turc, Congolais, } \\
\hline Agérien, Marocain et Tunisien & $23 \%$ & $28 \%$ & $21 \%$ & $15 \%$ \\
\hline \multicolumn{5}{|l|}{ Alemand, Danois, Français, } \\
\hline \multirow{2}{*}{\multicolumn{5}{|c|}{$\begin{array}{l}\text { Britannique, Luxembourgeois, } \\
\text { Néerlandais, Irlandais Suisse, }\end{array}$}} \\
\hline & & & & \\
\hline Etats-unien et Japonais & $8 \%$ & $5 \%$ & $10 \%$ & $12 \%$ \\
\hline tous les étrangers & $57 \%$ & $63 \%$ & $51 \%$ & 47\% \\
\hline
\end{tabular}

Source : INS, recensement de 1991, calculs personnels identifiable parmi l'ensemble des flux intra-urbains dirigés depuis ou vers Saint-Gilles au début des années 1990 : des populations au bas de l'échelle sociale, familles d'origine immigrée particulièrement, y laissent la place à des catégories plus favorisées, surtout composées de jeunes adultes formant des petits ménages sans enfant. Le caractère récent de la mise en place de ce schéma au début des années 1990 est un élément fort pour tisser le lien entre ce remplacement de populations et les dynamiques de rénovation urbaine qui se sont développées au cours des années 1980 à Saint-Gilles 9 .

Une étude ponctuelle menée au début des années 1990 renforce ce constat (De Lannoy \& Geets, 1994). Leurs auteurs ont analysé les transformations d'un ensemble de 85 logements rénovés à l'aide de subventions publiques à Saint-Gilles. Les contrastes observés entre le profil des habitants occupant ces logements avant et après leur rénovation rejoignent tout à fait les éléments mis en évidence ci-dessus : remplacement de familles avec enfants par des jeunes adultes vivant seul ou en couples, contraction des effectifs de nationalités à bas revenus et rehaussement des statuts socioprofessionnels. Selon les auteurs, il ne fait pas de doute que la hausse des loyers consécutive à la rénovation des logements analysés ait été la cause principale de l'éviction des anciens locataires. Ils n'excluent pas non plus la volonté de certains propriétaires de se « débarrasser » de locataires étrangers.

Plusieurs éléments indiquent enfin que la dynamique décrite ci-dessus s'est poursuivie au-delà de 1991. En particulier, tout au long des années 1990, Saint-Gilles a vu son nombre de ménages augmenter fortement tandis que le nombre d'habitants y diminuait légèrement. Ceci est cohérent avec la poursuite d'un processus de 
remplacement de grands ménages d'origine immigrée par des petits ménages de jeunes adultes.

\section{Espaces refuges et plaque redistributrice : les cas de Molenbeek, d'Anderlecht et d'Ixelles}

Les cartes des bilans migratoires des communes les plus réceptrices ou émettrices par rapport à Saint-Gilles apportent d'autres éclairages sur les dynamiques en jeu. Ixelles est sans conteste le principal pourvoyeur d'immigrants à Saint-Gilles entre 1990 et 1991. A l'opposé, trois communes ont un bilan particulièrement positif avec SaintGilles: Molenbeek (194 immigrants en provenance de Saint-Gilles, solde: +83), Anderlecht $(313,+125)$ et Bruxelles-Ville $(449,+89)$. La grande taille de cette dernière rend inadéquate la méthode utilisée. J'ai donc choisi de cartographier les bilans migratoires par rapport à Molenbeek et à Anderlecht, d'une part et par rapport à Ixelles, d'autre part.

\section{Molenbeek et Anderlecht}

Les cartes de Molenbeek et d'Anderlecht présentent une spectaculaire dichotomie de part et d'autre de l'axe du canal (figure 5). A de rares exceptions près, les bilans positifs ne se rencontrent qu'à l'ouest de cet axe. Il s'agit vraisemblablement de migrations centrifuges de populations (belges ou étrangères) inscrites dans le cadre d'une ascension sociale et/ou d'une fixation familiale. Ces ménages rechercheraient, dans le même quadrant de la ville, des espaces où l'environnement urbain est plus conforme aux attentes de familles avec enfants et où le marché du logement, de meilleure qualité qu'à Molenbeek ou à Anderlecht, leur est accessible (ce qui n'est pas le cas dans la partie orientale de la ville, plus aisée). Dans le cas des populations d'origine immigrée, ces dynamiques s'inscrivent dans un contexte d'allongement de la durée de séjour, voire d'abandon de l'idée du retour dans le pays d'origine (Mistiaen, Meert \& Kesteloot, 1995). Les bilans très négatifs de Koekelberg (surtout par rapport à Molenbeek) ne contredisent pas cette interprétation si l'on prend en compte l'effet des politiques de tracasseries administratives à l'encontre de familles d'origine immigrée menées dans cette commune. 
Figure 5. Molenbeek : bilans migratoires 1990-1991 ; Anderlecht : bilans migratoires 1990-1991.

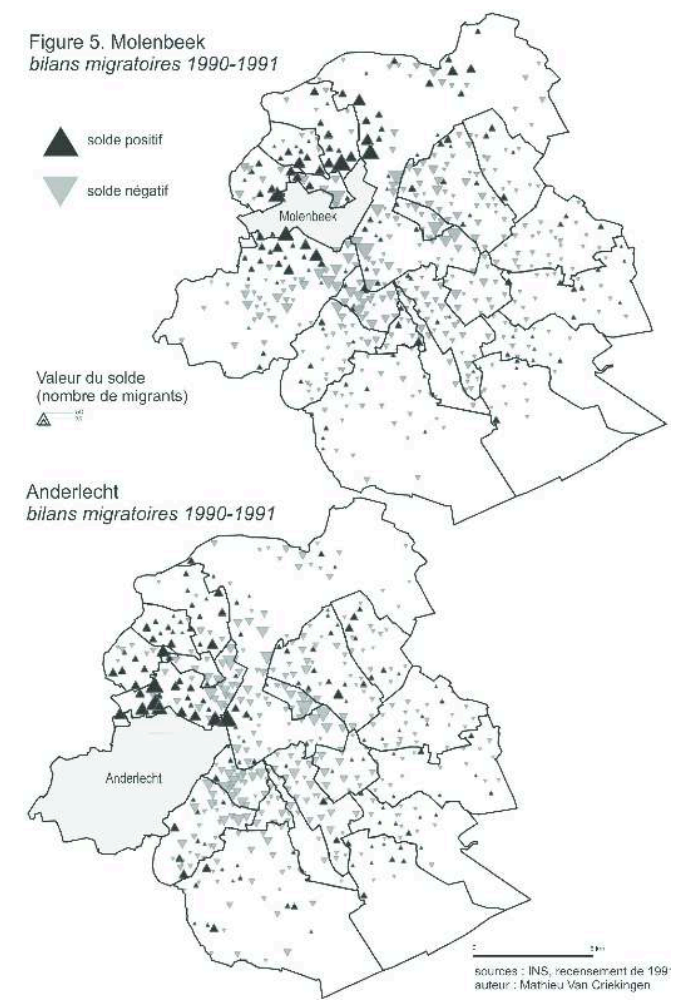

A l'est du canal, les bilans les plus négatifs ressortent dans le Pentagone et en première couronne. Ce canevas spatial était déjà en place au début des années 1980 mais présentait une dichotomie est / ouest moins prononcée (cartes non reprises). Il suggère que Molenbeek et Anderlecht fonctionneraient, dans une proportion non négligeable, comme « lieux d'accueil en dernier recours » de populations confrontées à la pression croissante sur le marché du logement dans les quartiers centraux. Les populations socialement fragilisées trouvent dans le vieux-Molenbeek ou à Cureghem à Anderlecht un tissu dense d'associations qui peuvent les aider à se loger et des opportunités plus ou moins informelles d'activité, souvent à base ethnique. L'insertion de ses populations dans le parc bâti passe le plus souvent par la location d'un logement de piètre qualité, sous-adapté à la taille du ménage, dans un bâtiment scindé en plusieurs appartements. Les propriétaires-bailleurs trouvent là l'opportunité de prolonger à bon compte la rentabilité de leur patrimoine en consentant un minimum d'investissement d'entretien ou de rénovation.

\section{Ixelles}

La carte des bilans migratoires par rapport à Ixelles entre 1990 et 1991 présente un tout autre visage que les deux précédentes (figure 6). Cette commune affiche le solde le plus négatif avec l'ensemble des autres communes bruxelloises (-1199). Cela se traduit sur la carte par la quasi-omniprésence des triangles orientés vers le haut. Ixelles est également la commune bruxelloise qui, proportionnellement à son nombre d'habitants, a reçu le plus d'émigrants en provenance du reste de la Belgique entre 1990 et 1991 . Ces éléments poussent à voir Ixelles comme la principale porte d'entrée sur le marché du logement bruxellois et comme principale plaque redistributrice vers les autres communes bruxelloises. La localisation dans cette commune des campus de l'ULB et de 
la VUB (environ 25000 étudiants) constitue sans nul doute un élément majeur d'explication de cet état de fait. Ixelles parait être le point de départ de l'itinéraire résidentiel autonome d'un grand nombre de jeunes adultes qualifiés (ou en voie de l'être) au sein de l'espace urbain bruxellois.

Figure 6. Ixelles : bilans migratoires 1990-1991 et 1980-1981.

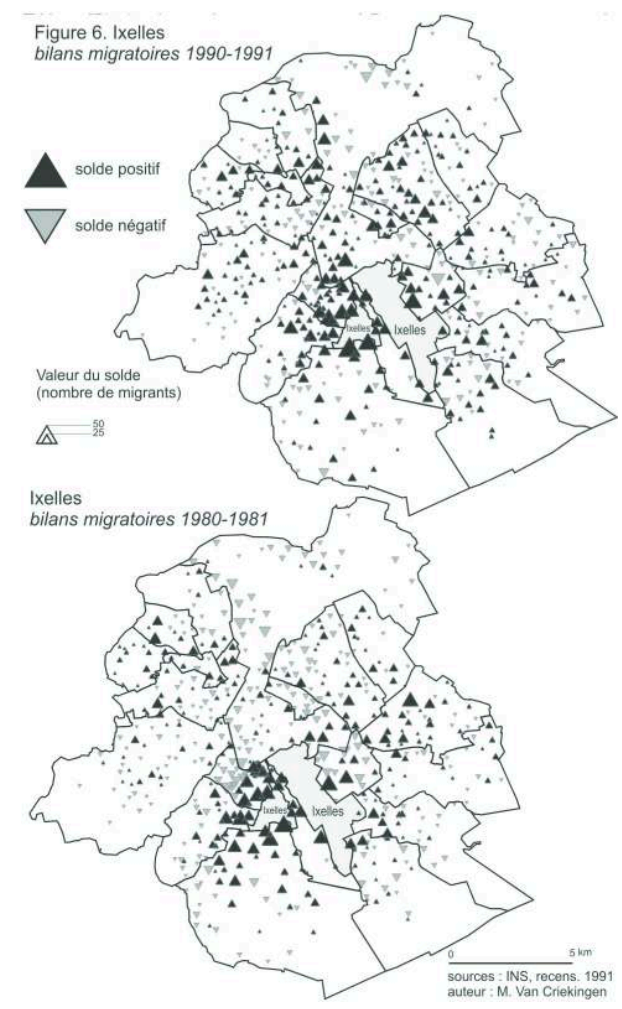

Les bilans les plus positifs par rapport à Ixelles ne sont pas pour autant équitablement répartis dans la ville. La carte de 1990-1991 gagne à cet égard à être comparée à celle portant sur la période 1980-1981. Entre les deux périodes, l'évolution est claire dans la partie médiane de Saint-Gilles, dans la quasi-totalité du Pentagone (au Sablon et dans les Marolles en particulier) et au sud du parc Josaphat. Il s'agit d'espaces qui ont vu se déployer des dynamiques de rénovation résidentielle pendant les années 1980 (Van Criekingen, à paraître). Les bilans restent par contre presque inchangés et presque nuls dans le vieux-Molenbeek et à Cureghem. Cette observation confirme l'idée d'espaces tenus à l'écart des itinéraires résidentiels des jeunes adultes qualifiés et qui fonctionnent autrement. Enfin, plusieurs bilans très positifs sont apparus au nordouest de la Région, à Laeken notamment. Ceci fait penser à des migrations de familles d'origine immigrée depuis le haut d'Ixelles, du quartier africain de Matongé notamment. Des études ciblées de ce quartier ont fait ressortir deux types de logiques : des migrations de familles d'origine immigrée en ascension sociale et en quête de meilleures conditions d'habitat ainsi que des déménagements contraints par l'accentuation de la pression immobilière induite par le marché des bureaux et certaines opérations de rénovation de logements (Mashini, 1998 ; Mwanza, 2000). 


\section{Le cas d'Etterbeek : d'autres évictions}

xelles, plusieurs études ont mis en évidence la pression exercée par le développement des activités tertiaires sur la fonction résidentielle, particulièrement eu égard à la présence des institutions européennes (Mens en Ruimte, 1992; De Keersmaecker et Carton, 1995 p. ex.). Cette pression est nourrie par la transformation d'immeubles résidentiels en surface de bureaux ou par l'insertion sur le marché du logement bruxellois de résidents étrangers dotés d'un pouvoir d'achat nettement supérieur à celui de la population autochtone (des fonctionnaires de l'U.E. p. ex.). Ces mécanismes alimentent un autre type de rénovation urbaine que celle rencontrée à Saint-Gilles.

La localisation des actifs reliés aux activités tertiaires internationales épouse globalement celle des franges aisées bruxelloises (au sein de la Région, préférence pour le quadrant sud-est) mais témoigne également de leur plus forte propension à résider dans la partie centrale de la ville. La partie orientale de la première couronne (les quartiers des Squares, du Cinquantenaire, de la place Jourdan ou l'axe de l'avenue Louise et les abords de la place du Châtelain) apparaît particulièrement attractive pour les actifs étrangers à hauts revenus reliés aux fonctions internationales de Bruxelles. Extension bourgeoise du XIXe siècle, cette partie de la ville offre à la fois un cadre bâti d'une grande qualité architecturale, un accès aisé à la centralité métropolitaine et la proximité des principaux sièges des fonctions internationales (Christiaens, Claes \& Deroo, 1998 ; Bastin, 2001). Dans ce cadre, il est intéressant de se pencher sur le cas d'Etterbeek, commune de petite taille située dans la partie orientale de la première couronne, à proximité immédiate du principal site d'implantation des institutions européennes.

\section{Etterbeek : flux migratoires entre 1990 et 1991}

La carte des bilans migratoires par rapport à Etterbeek ne montre pas de logique de quadrant (figure 7). En effet, les bilans positifs sont d'importance similaire dans la partie d'Ixelles qui borde Etterbeek, dans l'axe de la chaussée de Wavre à Auderghem (axe de standing moyen dans le quadrant sud-est aisé), dans l'est de Schaerbeek mais aussi dans l'ouest de la Région, au-delà du croissant pauvre (à Anderlecht, Koekelberg, Jette). Il s'agit d'espaces sociologiquement proches d'Etterbeek (domination des classes moyennes belges) mais où le marché du logement est moins tendu. A contrario, les soldes négatifs ou quasi nuls affichés par l'essentiel du croissant pauvre témoignent de la très faible attractivité de ces espaces pour les émigrants en provenance d'Etterbeek et, à l'opposé, de l'inaccessibilité du marché du logement etterbeekois pour des ménages à revenus modestes. Comme pour Saint-Gilles, ce canevas spatial a été mis en place au cours des années 1980. La carte des bilans migratoires par rapport à Etterbeek pour la période 1980-1981 montre un aspect beaucoup plus ramassé sur le quadrant oriental de la Région. Les émigrations vers la seconde couronne aisée dominaient. 
Figure 7. Etterbeek : bilans migratoires 1990-1991 et 1980-1981.

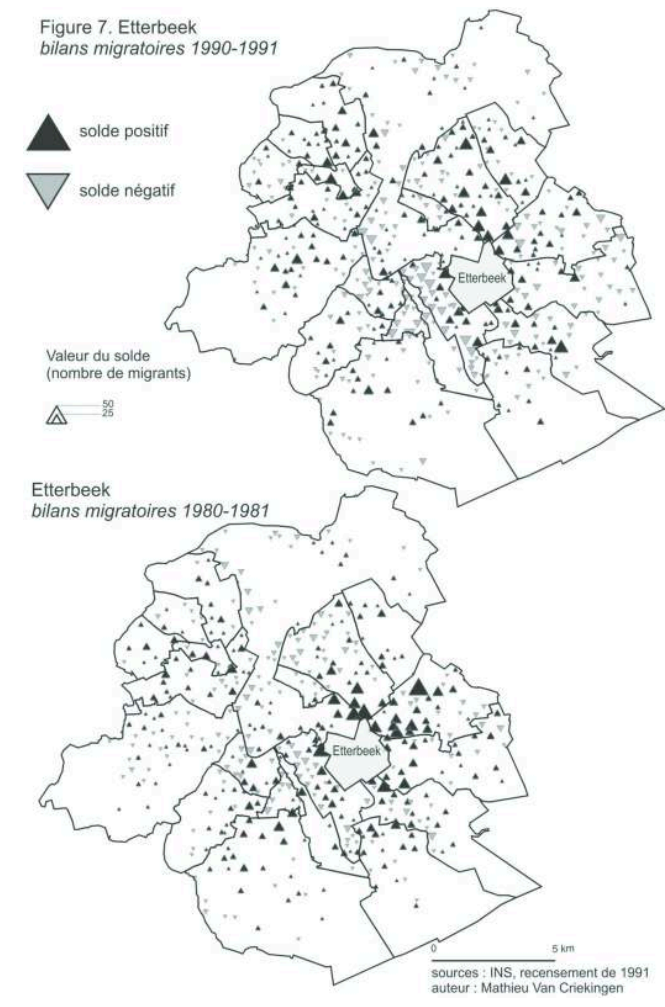

\section{Etterbeek : contrastes démographiques}

31 Comme dans le cas saint-gillois, la pyramide d'âges de l'ensemble des migrants de ou vers Etterbeek fait ressortir les classes d'âges les plus migrantes (figure 8). Il est donc à nouveau impératif de décomposer ces flux afin de percer l'écran des flux croisés de populations. Ceci a été réalisé de la même manière que dans le cas de Saint-Gilles. 
Figure 8. Structure d'âges des migrants par rapport à Saint-Gilles.

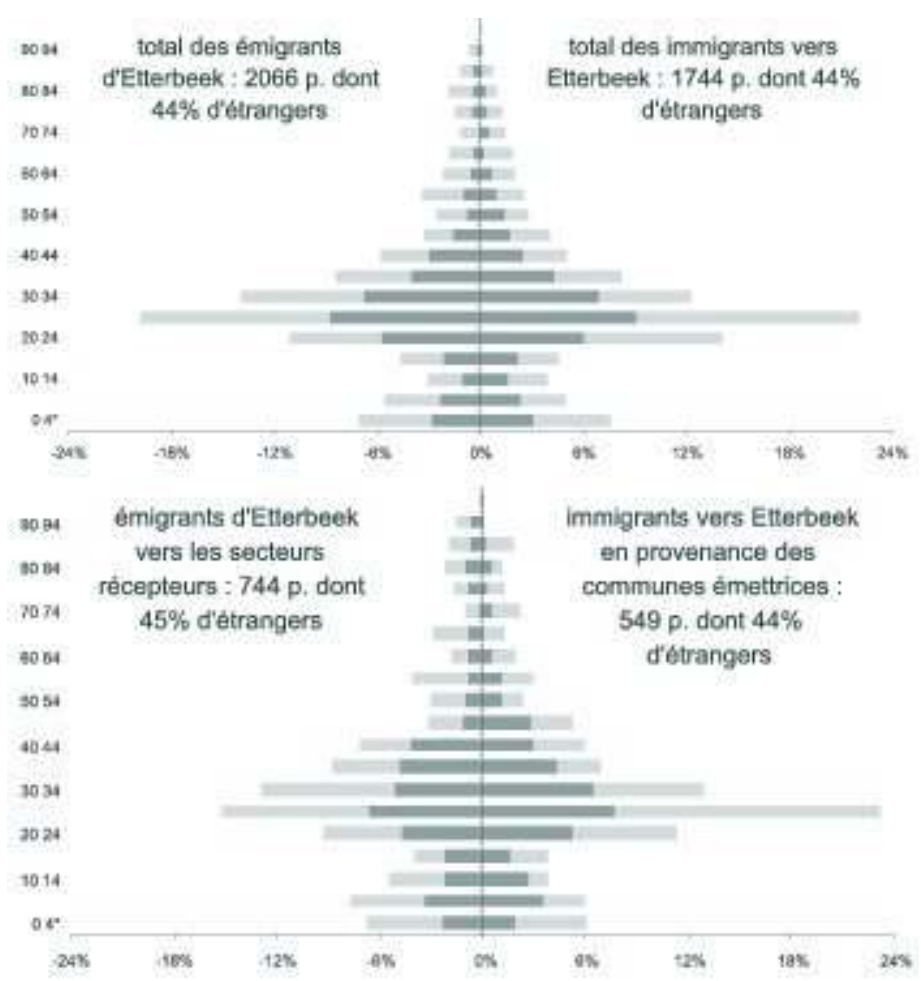

Les Belges à la naissance sont en clair, les étrangers, y compris les naturalisés, sont en foncé. Note: cf. figure 4.

Les émigrants d'Etterbeek vers les secteurs statistiques où le bilan est supérieur ou égal à 5 (744 personnes, $36 \%$ des émigrants de cette commune) sont dominés par les Belges (de naissance). Trois groupes d'âges d'ordinaire peu migrants sont plus représentés qu'au sein de l'ensemble des émigrants d'Etterbeek: les 5-14 ans, les 40-64 ans et les plus de 80 ans. Par ailleurs, j'ai retenu trois communes parmi celles qui ont un bilan négatif avec Etterbeek: Ixelles (358 émigrants vers Etterbeek, solde : -83), WoluwéSaint-Pierre $(139,-11)$ et Saint-Josse $(52,-6)$. Elles regroupent $31 \%$ des émigrations vers Etterbeek ${ }^{10}$. La structure par âges de ces immigrants à Etterbeek met en évidence une nette surreprésentation des jeunes adultes (les Belges âgés entre 25 et 29 ans en particulier) et un nombre restreint d'enfants. Ceci évoque, comme pour Saint-Gilles, l'arrivée de " post-étudiants », en provenance d'Ixelles surtout.

Plus encore que parmi ceux qui s'installèrent à Saint-Gilles entre 1990 et 1991, les universitaires dominent l'ensemble des immigrants à Etterbeek provenant des communes émettrices (tableau 2). Le décalage vers les faibles qualifications des émigrants vers les secteurs récepteurs n'est, quant à lui, pas du tout rencontré. Ce sont cette fois les diplômés du secondaire supérieur qui dominent ce groupe de migrants. La même image de domination des profils "moyens" parmi les émigrants d'Etterbeek vers les secteurs récepteurs se dégage de la structure par types de profession. En effet, les employés de bureau dominent la répartition alors qu'ils étaient trois fois moins représentés que les professions manuelles chez ceux qui quittèrent Saint-Gilles pendant la même période. Les émigrants de ces deux communes se distinguent encore par leur pourcentage de chômeurs (17 \% pour Etterbeek, 26 \% pour Saint-Gilles). 
Tableau 2. Caractéristiques socio-économiques des migrants de ou vers Etterbeek entre 1990 et 1991.

\begin{tabular}{|c|c|c|c|c|}
\hline & \multicolumn{2}{|c|}{$\begin{array}{l}\text { MIGRANTS } \\
\text { DEPUIS ETTRBEEKK }\end{array}$} & \multirow{2}{*}{$\begin{array}{l}\begin{array}{l}\text { IMMIGRANTS } \\
\text { VERS ETTERBEEK }\end{array} \\
\text { total }\end{array}$} & \multirow[b]{2}{*}{$\begin{array}{l}\text { depuis les } \\
\text { communes } \\
\text { au bilan } \leq-5\end{array}$} \\
\hline & total & $\begin{array}{l}\text { vers les } \\
\text { sect. stat. } \\
\text { au bilan } \geq 5\end{array}$ & & \\
\hline \multicolumn{5}{|c|}{ plus haut diplatome obtenu (\% dans la populatation active) } \\
\hline universitaire & $17 \%$ & $17 \%$ & $21 \%$ & $28 \%$ \\
\hline enseignement supérieur & $14 \%$ & $16 \%$ & $15 \%$ & $15 \%$ \\
\hline secondaire supérieur & $23 \%$ & $24 \%$ & $18 \%$ & $15 \%$ \\
\hline secondaire inférieur & $18 \%$ & $16 \%$ & $19 \%$ & $18 \%$ \\
\hline primaire & $7 \%$ & $7 \%$ & $6 \%$ & $6 \%$ \\
\hline sans diplôme et inconnu & $20 \%$ & $20 \%$ & $21 \%$ & $18 \%$ \\
\hline \multicolumn{5}{|c|}{ type de profession (\% dans la population active occupée) } \\
\hline $\begin{array}{l}\text { professions scientifiques, } \\
\text { liberrales et assimiles }\end{array}$ & $19 \%$ & $17 \%$ & $23 \%$ & $23 \%$ \\
\hline $\begin{array}{l}\text { chefss 'entrepprse et } \\
\text { cadres superieurs }\end{array}$ & $10 \%$ & $10 \%$ & $10 \%$ & $9 \%$ \\
\hline employés de bureau & $22 \%$ & $24 \%$ & $18 \%$ & $18 \%$ \\
\hline commerçants & $9 \%$ & $8 \%$ & $11 \%$ & $11 \%$ \\
\hline $\begin{array}{l}\text { Ouvriers, artisans, techniciens } \\
\text { et professions des transports }\end{array}$ & $21 \%$ & $23 \%$ & $22 \%$ & $23 \%$ \\
\hline travalleurs des services & $10 \%$ & $12 \%$ & $11 \%$ & $10 \%$ \\
\hline inconnus et autres & $8 \%$ & $6 \%$ & $6 \%$ & $5 \%$ \\
\hline \multicolumn{5}{|l|}{ nationalité á la naissance } \\
\hline $\begin{array}{l}\text { ex-Yougoslave, Turc, Congolais, } \\
\text { Agérien, Marocain et Tunisien }\end{array}$ & $16 \%$ & $16 \%$ & $15 \%$ & $15 \%$ \\
\hline \multicolumn{5}{|l|}{ Alemand, Danois, franģais, } \\
\hline Néerlandais, Irlandais, Suisse. & & & & \\
\hline Etats-unien et Japonais & $11 \%$ & $7 \%$ & $12 \%$ & $14 \%$ \\
\hline tous les etrangers & $44 \%$ & $45 \%$ & $44 \%$ & $44 \%$ \\
\hline
\end{tabular}

Source : INS, recensement de 1991, calculs personnels

Comme à Saint-Gilles, les nationalités représentatives des actifs occupés dans les fonctions tertiaires internationales sont plus représentées parmi les immigrants que parmi les émigrants. Néanmoins, le cas d'Etterbeek se distingue clairement de celui de Saint-Gilles par une part nettement plus faible d'émigrants de nationalités représentatives des travailleurs immigrés vers les secteurs récepteurs (16\% contre $28 \%)$.

L'ensemble de ces éléments révèle un schéma bien différent de celui mis en évidence pour Saint-Gilles. Si une partie des émigrants d'Etterbeek sont à qualifier d'évincés, il s'agirait avant tout de classes moyennes belges. Une interprétation possible est que, face à la pression croissante sur le marché immobilier, ces ménages quitteraient Etterbeek pour s'installer dans des espaces semblables au plan sociologique mais où le marché immobilier est moins tendu. Le terme "d'évincé » serait donc à nuancer dans ce cas-ci, d'autant plus que la pression immobilière induite par le développement tertiaire international dans la partie orientale de la première couronne implique, pour des ménages propriétaires de leur logement, des opportunités de revendre leur bien à un très bon prix.

\section{Conclusion}

Malgré les limites qu'imposent les données utilisées, l'analyse des migrations intraurbaines à Bruxelles au début des années 1990 fait ressortir différents processus de remplacement de populations parmi l'ensemble des mouvements migratoires au sein de la ville. L'analyse appuie l'idée d'un lien étroit entre ces processus et différentes 
dynamiques de rénovation urbaine. En particulier, rénovation et surconcentration de populations socialement fragilisées paraissent s'articuler dans certains cas comme les deux faces d'une même pièce.

Alors que nombre de discours projettent des dynamiques de rénovation une image uniformément positive (d'où l'usage de termes enthousiastes tels que " revitalisation », « renaissance », «renouveau »,...), celles-ci contribuent à aggraver le fractionnement socio-spatial de l'espace urbain, ce qui ne peut qu'aggraver les tensions sociales dans la ville. Ces dynamiques vont par conséquent à l'encontre d'un modèle de développement urbain socialement durable, à moins qu'elles ne soient efficacement encadrées par des politiques d'amélioration structurelle de la position des populations socialement fragilisées sur le marché du logement urbain.

\section{BIBLIOGRAPHIE}

ATKINSON R. (2000), « Measuring gentrification and displacement in Greater London », Urban Studies, 37, 1, pp. 149-165.

BASTIN S. (2001), Géographie des étrangers haut-statut à Bruxelles, mémoire de licence, Laboratoire de géographie humaine, Université Libre de Bruxelles, non publié.

BOKHORST H. (2000), « La chasse aux pauvres est ouverte ! », Le Soir, 13 septembre.

CHRISTIAENS E., CLAES A. \& DEROO R. (1998), L'impact socio-économique des institutions européennes et internationales dans la Région de Bruxelles-Capitale, Bruxelles, IRIS Consulting.

DE KEERSMAECKER M.-L. \& CARTON L. (1995), Le droit au logement. Bilan d'une première législature bruxelloise, Bruxelles, Editions Vie Ouvrière.

DE LANNOY W. \& DE CORTE S. (1994), « De migraties van Marokkanen en Turken binnen het Brusselse Gewest in de periode 1988-1992 », in VAN HECKE E. \& GOOSSENS M. (éd.), Acta Geographica Lovaniensia : Liber Amicorum Herman Van der Haegen, 34, pp. 63-69.

DE LANNOY W. \& GEETS A. (1994), « Urban renewal in Brussels : how and for whom? », Revue Belge de Géographie, 55, 1-2, pp. 23-30.

DECROLY J.-M., VAN CRIEKINGEN M. \& CRAHAY A. (2001), « Les quartiers en crise face aux nouvelles politiques urbaines » in DEVUYST D., HENS L., IMPENS R. (éd.), Neighbourhoods in crisis and sustainable urban development, VUB University Press, pp. 39-62.

DELBART V. (1999), La gentrification à Bruxelles et ses conséquences sur les populations les plus démunies, rapport de travaux pratiques, Laboratoire de géographie humaine, Université Libre de Bruxelles, non publié.

EGEC (Etats Généraux de l'Ecologie Communale) (2000), Pour un développement durable des quartiers, Actes du forum du 28 juin 2000, Groupe Ecolo au Conseil régional bruxellois.

LEGATES R. T. \& HARTMAN C. (1986) « The anatomy of displacement in the United States », in SMITH N. \& WILLIAMS P. (eds.), Gentrification of the city, Winchester, Allen \& Unwin, pp. 178-200. 
LYONS M. (1996), « Gentrification, socioeconomic change, and the geography of displacement », Journal of Urban Affairs, 18, 1, pp. 39-62.

MARISSAL P. (1994), « Rénovation urbaine et mutations sociales dans les quartiers défavorisés de Bruxelles », Revue Belge de Géographie, 55, 1-2, pp. 83-92.

MASHINI D. M. M. (1998), «L'intégration socio-économique de la population originaire d'Afrique noire dans la Région de Bruxelles-Capitale », Revue Belge de Géographie, 63, 1, pp. 55-70.

MENS EN RUIMTE asbl (1992), L'impact des institutions européennes à Bruxelles, Bruxelles.

MISTIAEN P., MEERT H. \& KESTELOOT C. (1995), « Polarisation socio-spatiale et stratégies de survie dans deux quartiers bruxellois ", Espace Populations Société, 3, pp. 277-290.

MWANZA H. (2000), « Adieu Matongé ? », C4, 71, Bruxelles.

NOEL F. (1998), La ville rapiécée. Les stratégies de la réhabilitation à Bruxelles, Bruxelles, Editions de l'Université de Bruxelles.

RBDH (Rassemblement Bruxellois pour le Droit à l'Habitat) (2001), Droit au logement pour tous, texte de plate-forme.

SMITH N. (1996), The new urban frontier. Gentrification and the revanchist city, London, Routledge.

SMITH N. \& WILLIAMS P. (eds.) (1986), Gentrification of the city, Winchester, Allen \& Unwin.

VAN CRIEKINGEN (2001), La rénovation résidentielle à Montréal et à Bruxelles, thèse de doctorat, Laboratoire de géographie humaine, Université Libre de Bruxelles, non publié.

VAN CRIEKINGEN (à paraître), « La ville revit ! Formes, politiques et impacts de la revitalisation résidentielle à Bruxelles » in BIDOU C., HIERNAUX D. et RIVIERE D’ARC H. (éd.), Retours en ville : approches comparées des processus de gentrification urbaine : Europe, Amériques, Paris, Descartes et Cie.

\section{NOTES}

1. La Région de Bruxelles-Capitale est composée de 19 communes dotées de pouvoirs propres. Pour l'usage statistique, chaque commune est découpée en secteurs statistiques (722 au total à Bruxelles). La taille des secteurs statistiques correspond grosso modo à celle d'un petit quartier.

2. La nature de ces derniers a été étudiée en détail par ailleurs, dans le cas de Bruxelles notamment (Van Criekingen, 2001, à paraître).

3. Les niveaux de qualification retenus sont: sans diplôme/diplôme primaire/secondaire inférieur/secondaire supérieur/enseignement supérieur.

4. Le solde est réparti entre 16300 entrées depuis le reste de la Belgique et 19200 entrées depuis l'étranger (INS, recensement de 1991, calcul personnel).

5. Pour représenter la classe des 0-4 ans, j'ai estimé un nombre théorique de migrants de moins d'un an en divisant simplement par 4 l'effectif de migrants âgés de 1 à 4 ans.

6. Les nationalités inconnues sont comprises dans les étrangers.

7. Après quelques essais, le seuil a été fixé à 5 .

8. En Belgique, les résidents de nationalité étrangère hors Union Européenne n'ont pas le droit de vote.

9. Aucun des chiffres présentés ne tient évidemment compte des personnes en situation irrégulière. Il s'agit de groupes particulièrement vulnérables sur le marché du logement (les sanspapiers p. ex.) (RBDH, 2001). Or, à Saint-Gilles, il est très vraisemblable que les transformations récentes des abords de la gare du Midi aient mené à l'expulsion d'un nombre important d'entre eux. 
10. Les autres communes ont un bilan soit positif avec Etterbeek, soit quasiment nul. BruxellesVille a un bilan très faiblement négatif étant donné sa taille (-9) et pose problème dans le cadre de la méthode adoptée. Je ne l'ai donc pas retenue.

\section{RÉSUMÉS}

L'analyse des mouvements migratoires intra-urbains à Bruxelles au début des années 1990 met en évidence différents processus de remplacement de populations parmi l'ensemble des mouvements migratoires qui se déploient au sein de la ville. L'analyse appuie l'idée d'un lien étroit entre ces processus et différentes dynamiques de rénovation urbaine. Ainsi, à l'échelle de l'ensemble de la ville, rénovation et surconcentration spatiale de populations socialement fragilisées dans les portions déjà les plus dégradées et paupérisées de la ville paraissent s'articuler souvent comme les deux faces d'une même pièce. Les dynamiques de rénovation urbaine contribuent de la sorte à aggraver le fractionnement socio-spatial de la ville et, par conséquent, vont à l'encontre d'un modèle de développement urbain socialement durable.

The analysis of intra-urban migration in Brussels at the beginning of the 1990s brings to the fore different processes of population replacement among the whole range of migration flows taking place within the city. I argue that these processes are closely related to different types of urban renewal. In some cases, urban renewal and increasing spatial concentration of the urban poor in already most decayed and impoverished central neighbourhoods seems to go hand in hand. Processes of urban renewal reinforce in that way the socio-spatial polarisation of the city and, therefore, run counter to a model of socially sustainable urban development.

\section{INDEX}

Keywords : urban renewal, gentrification, population replacement, intra-urban migrations, Brussels

Mots-clés : rénovation urbaine, gentrification, remplacement de population, migrations intraurbaines, Bruxelles

\section{AUTEUR}

\section{MATHIEU VAN CRIEKINGEN}

Institut de Gestion de l'Environnement et d'Aménagement du Territoire, Université Libre de

Bruxelles, mvancrie@ulb.ac.be 